3 THE IMPACT OF MENTAL HEALTH AND WELLBEING ON GCSE PERFORMANCE IN ENGLAND: A LONGITUDINAL ANALYSIS OF THE NATIONAL PUPIL DATABASE LINKED TO UNDERSTANDING SOCIETY

NR Smith*, L Marshall, M Albakri, R Sehmi. Centre for Children and Families, National Centre for Social Research, London, UK

\subsection{6/bmjpo-2019-RCPCH-SAHM.3}

Aim To evaluate prospectively the influence of mental health and wellbeing in early adolescence on educational attainment.

Methods Using the data linkage between 1073 participants from the UK Household Longitudinal Study (2009-2012) and the National Pupil Database, we investigate longitudinal associations between mental health at ages 11-14 and later attainment at GCSE. We use the term mental health to encompass socio-emotional development (measured using a self-completed Strengths and Difficulties Questionnaire) and subjective wellbeing (measured using a self-assessment of happiness with life as a whole). Young people were scored as having typical (014), borderline 15-17), or atypical (18-40) levels of socio-emotional development. Happiness was defined as a score of 1-3 on a 7 point scale.

Results Low levels of socio-emotional development aged 11-14 were associated with a lower likelihood of gaining $5 \mathrm{~A}^{*}-\mathrm{C}$ GCSEs. Over half (57\%) of young people with low levels and almost half $(46 \%)$ of those with borderline low levels of socio-emotional development did not achieve $5 \mathrm{~A} * \mathrm{C}$ GCSEs including maths and English, compared to 30\% of their peers with typical levels of development. This association remained significant after controlling for individual demographic and household social and economic factors, (low SDQ odds ratio: 2.80, [1.95,4.03]; (Borderline SDQ odds ratio: 1.94, [1.31,2.87]). Wellbeing at ages $11-14$ was positively associated with later GCSE attainment. Almost half (48\%) of children who were not happy with their life as a whole at ages 11-14 did not achieve $5 \mathrm{~A}^{*}$ to C GCSEs, compared to around onethird $(35 \%)$ of their happier counterparts. This association was significant after controlling for individual demographic and household social and economic factors (odds ratio: 1.59, [1.09-2.32]).

Conclusion Mental health at ages 11-14 was independently linked to educational success at age 16. Given the known links between educational attainment and later life outcomes, this evidence illuminates an important mechanism through which poor health in childhood and early adolescence can limit young people's life chances. It provides another strong case for investing in adolescent mental health in addition to the health and economic cases widely evidenced in the literature.

\section{$4 \quad$ BOYS, BULK, AND BODY IDEALS: SEX DIFFERENCES IN WEIGHT GAIN ATTEMPTS AMONG ADOLESCENTS IN THE UNITED STATES}

\footnotetext{
${ }^{1} \mathrm{JM}$ Nagata*, ${ }^{2} \mathrm{~K}$ Bibbins-Domingo, ${ }^{1} \mathrm{AK}$ Garber, ${ }^{3} \mathrm{~S}$ Griffiths, ${ }^{2} \mathrm{E}$ Vittinghoff, ${ }^{4} \mathrm{SB}$ Murray. ${ }^{1}$ Division of Adolescent and Young Adult Medicine, Department of Pediatrics, University of California, San Francisco, USA; ${ }^{2}$ Department of Epidemiology and Biostatistics, University of California, San Francisco, USA; ${ }^{3}$ Melbourne School of Psychological Sciences, University of Melbourne, Melbourne, Australia; ${ }^{4}$ Department of Psychiatry, University of California, San Francisco, USA
}

10.1136/bmjpo-2019-RCPCH-SAHM.4
Aim Research on attempts to gain weight among adolescent males is limited and has not yet been examined using nationally representative samples in the United States. The objective of this study was to estimate the prevalence of weight gain attempts in adolescent boys using a nationally representative sample and to examine differences in weight gain attempts by weight classification, weight self-perception, age, race/ethnicity, and sexual identification.

Methods Participants were 15,624 high school students from the nationally representative 2015 Youth Risk Behavior Survey.

Results Overall, 29.6\% of adolescent boys reported attempts to gain weight, including $39.6 \%$ of boys who were normal weight, $12.8 \%$ who were overweight, and $10.6 \%$ who were obese by body mass index (BMI). In contrast, only $6.5 \%$ of adolescent girls reported attempts to gain weight. Although only $3.3 \%$ of adolescent males are underweight by BMI, $19.3 \%$ perceive themselves to be underweight. Further, over half of adolescent males who are overweight by BMI perceive themselves to be about the right weight. African American (Odds ratio [OR] 1.89; 95\% confidence interval [CI] 1.502.38) and mixed race (OR 1.62; 95\% CI 1.16-2.26) adolescent males had greater odds of weight gain attempts than White adolescent males. Adolescent males identifying as bisexual had lower odds (OR 0.47; 95\% CI 0.25 - 0.88) of weight gain attempts than adolescent males identifying as heterosexual.

Conclusions Weight gain attempts are common among adolescent boys including those who are considered normal weight, overweight, or obese by BMI; African American or mixed race; and those self-identifying as heterosexual. Consideration of the unique nature of male body image, particularly adolescent boys' perceptions of their own weight and weight-gain attempts, should be incorporated into primary care screening for adolescent boys.

\section{INTERNATIONAL COMPARISONS OF HEALTH AND WELLBEING IN ADOLESCENCE AND EARLY ADULTHOOD}

R Shah*, A Hagell. Research, Association for Young People's Health, London, UK

\subsection{6/bmjpo-2019-RCPCH-SAHM.5}

Aim To provide a snapshot of how the UK compares internationally with similar high-income countries in relation to young people's (YP's) health measures.

Methods Undertaken in partnership with The Nuffield Trust, AYPH undertook a descriptive study comparing health and wellbeing indicators for YP in the UK to 18 other highincome countries. Publicly available international datasets were used for these analyses. Some of the 17 areas of comparison included obesity, long-standing illness, deprivation, adolescent birth rate, asthma death rate, diabetes, cancer mortality, smoking, alcohol consumption, overall mortality and transport injury death.

Results

- The UK has the highest rate of deaths from asthma for YP aged 10-24, compared to all European countries in the comparative group, and the fourth highest overall behind the USA, Australia and New Zealand.

- As well as having the highest rates of obesity in 15- to 19year-olds compared to the 14 European countries in the comparative group, the UK also has one of the greatest 